\title{
Modeling Spatial Spillovers of Divorce in Senegal Using Spatial Durbin Model: A Maximum Likelihood Estimation Approach
}

\author{
Alassane Aw*, Emmanuel Nicolas Cabral" \\ Laboratory of Mathematics and Applications, Assane Seck University of Ziguinchor, Ziguinchor, Senegal \\ Email address: \\ alassane.aw.ansd@gmail.com (A. Aw), encabral@univ-zig.sn (E. N. Cabral) \\ *Corresponding authors
}

\section{To cite this article:}

Alassane Aw, Emmanuel Nicolas Cabral. Modeling Spatial Spillovers of Divorce in Senegal Using Spatial Durbin Model: A Maximum Likelihood Estimation Approach. American Journal of Theoretical and Applied Statistics. Vol. 8, No. 1, 2019, pp. 1-6. doi: $10.11648 /$ j.ajtas.20190801.11

Received: December 16, 2018; Accepted: January 5, 2019; Published: January 24, 2019

\begin{abstract}
Spatial Durbin Model (SDM) is one of the family of spatial autoregressive models. In this paper, we use the SDM model to determine the spatial spillovers of divorce in Senegal. The variable of interest is the rate of divorce and the explanatory variables are the rate of illiteracy and the average age at marriage in Senegal. The model parameters are estimated by the maximum likelihood technique. The estimation of the autoregressive parameter is performed using numerical optimization of the concentrated log-likelihood of the SDM model. The results obtained showed that the rate of illiteracy and the average age at marriage have a real impact on the rate of divorce in Senegal. We also note that the departments of the country that are closed are more similar than the distant departments in relation to the divorce data. Direct and indirect effects were used to measure changes in the rate of divorce as a result of changes in the rate of illiteracy and the average age at marriage.
\end{abstract}

Keywords: Spatial Durbin Model, Maximum Likelihood Estimation, Spatial Spillovers, Impact Measures

\section{Introduction}

Legally speaking, divorce is the breaking of the conjugal bond pronounced by a judge. A divorce arranged by both spouses and their families without the intervention of the judge "is not valid in the sense of the law". There are two types of divorce: consent divorce and contentious divorce.

In Senegal, divorce cases are growing more and more. According to the National Agency of Statistics and Demography of Senegal, divorced people are more numerous in the region of Dakar (2.5\%). The region of Dakar is followed by the regions of Matam and Saint-Louis with $1.5 \%$ each (identical to the national average). The region of Sedhiou with $0.5 \%$ recorded the lowest proportion of divorced people, followed by Kedougou and Kaffrine with $0.7 \%$ each. However, we note that marriages are much more stable in Senegal than in many other African countries.

The causes of divorce are multiple. We can note, among others: the evolution of morals in Senegal, the secular status of the country, the incompatibility of the couple, the financial problems, the abusive behavior, the divergence of projects for the future, and so on.

Spatial linear models deal with spatial effects: spatial heterogeneity and spatial dependence. Spatial heterogeneity corresponds to a differentiation in effects across space. However, spatial dependence states that what happens in area $\mathrm{i}$ depends on what happens in area $\mathrm{j}[1]$.

We say that there are spatial spillovers when changes noted in one area cause changes in other areas [2-4]. We distinguish global spillovers to local spillovers. Global spillovers exist when changes noted in one area impact changes in all areas. Local spillovers occur when changes in one area only impact neighboring areas [5].

Several studies have focused on applications of the spatial Durbin model.

The spatial Durbin model was used to estimate functional coefficients and spatial weighting functions via a nonparametric two-stage least squares estimation [6]. It was also used to examine the role and mechanism of environmental regulation in the impact of green innovation on 
industrial green development in China [7]. In the context of dynamic panel models, the identification of the spatial Durbin model has been studied using 2SLS and ML estimations [8]. The spatial Durbin model allowed to study relationships between rate of illiteracy and percentage of households owning a mobile phone in East Java, Indonesia [9]. It also allowed to correct the bias of omitted variables in the specification of spatial regression models [10]. In the context of Bayesian estimation, the spatial Durbin model was used to study income convergence in Japan [11].

In section 2, we give the materials and methods which help us to model the spatial effects of divorce in Senegal. The results obtained are given in section 3. Finally, in Section 4, we discuss the results with an application of the spatial Durbin model to divorce's data.

\section{Materials and Methods}

The spatial Durbin model (SDM) for cross-sectional data is given by the following structural model:

$y=\alpha 1_{n}+\rho W y+X \beta+W X \gamma+\epsilon, \quad \epsilon \sim N\left(0, \sigma^{2} I_{n}\right)$

Where:

$y$ is a vector $n \times 1$ that collects the dependent variable for each spatial units;

$W$ is a $n \times n$ spatial weight matrix;

$X$ is a $n \times p$ matrix of independent variables;

$\rho$ is a parameter that measures the degree of spatial correlation in the dependent variable which is also called autoregressive parameter;

$\beta$ is a $p \times 1$ vector of parameters;

$\gamma$ is a $p \times 1$ vector of parameters that measures the degree of spatial correlation in the independent variables;

$\epsilon$ is a $n \times 1$ vector of error terms;

$1_{n}$ is a $n \times 1$ vector of ones;

$I_{n}$ is the identity matrix of order $n$;

$n$ is the number of observations or locations; and $p$ is the number of independent variables.

From equation (1), when $X=0$, we obtain the spatial autoregressive model in first order, such as:

$$
y=\alpha 1_{n}+\rho W y+\epsilon, \quad \epsilon \sim N\left(0, \sigma^{2} I_{n}\right)
$$

When $\rho=0$, we obtain the spatial lag X model given by:

$$
y=\alpha 1_{n}+X \beta+W X \gamma+\epsilon, \quad \epsilon \sim N\left(0, \sigma^{2} I_{n}\right)
$$

When $\gamma=0$, equation (1) will be spatial autoregressive model or mixed regressive-autoregressive model shown in:

$$
y=\alpha 1_{n}+\rho W y+X \beta+\epsilon, \quad \epsilon \sim N\left(0, \sigma^{2} I_{n}\right)
$$

When $\rho=0$ and $\gamma=0$, we obtain the general linear regression model given by:

$$
y=\alpha 1_{n}+X \beta+\epsilon, \quad \epsilon \sim N\left(0, \sigma^{2} I_{n}\right)
$$

The spatial Durbin model is a special case of the family of spatial autoregressive models with spatial lag on explanatory variables [12]. This model is important because spatial relationships are both noted in the dependent and the independent variables.

In the spatial Durbin model given in equation (1), $\alpha$ is intercept, $\beta$ is a vector of regression parameters and $\gamma$ is a weighted vector of regression parameter.

Let $Z=\left[\begin{array}{lll}1_{n} & X & W X\end{array}\right]$ and $\delta=\left(\begin{array}{lll}\alpha & \beta^{t} & \gamma^{t}\end{array}\right)^{t}$, then the spatial Durbin model can be written as:

$$
y=\rho W y+Z \delta+\epsilon, \quad \epsilon \sim N\left(0, \sigma^{2} I_{n}\right)
$$

If the autoregressive parameter $\rho$ satisfies $|\rho|<1$, then the matrix $I_{n}-\rho W$ becomes nonsingular [13]. Therefore, equation (6) will be:

$$
y=\left(I_{n}-\rho W\right)^{-1} Z \delta+\left(I_{n}-\rho W\right)^{-1} \epsilon, \quad \epsilon \sim N\left(0, \sigma^{2} I_{n}\right)
$$

The expected value of $y$ is given by:

$$
\begin{aligned}
\mathbb{E}(y \mid Z, W) & =\mathbb{E}\left(\left(I_{n}-\rho W\right)^{-1} Z \delta \mid Z, W\right)+\mathbb{E}\left(\left(I_{n}-\rho W\right)^{-1} \epsilon \mid Z, W\right)=\left(I_{n}-\rho W\right)^{-1} Z \delta+\left(I_{n}-\rho W\right)^{-1} \mathbb{E}(\epsilon \mid Z, W) \\
& =\left(I_{n}-\rho W\right)^{-1} Z \delta
\end{aligned}
$$

The variance-covariance matrix of $y$ is given by:

$$
\begin{aligned}
& \operatorname{Var}(y \mid Z, W)=\operatorname{Var}\left(\left(I_{n}-\rho W\right)^{-1} Z \delta+\left(I_{n}-\rho W\right)^{-1} \epsilon \mid Z, W\right)=\operatorname{Var}\left(\left(I_{n}-\rho W\right)^{-1} \epsilon \mid Z, W\right) \\
& =\left(I_{n}-\rho W\right)^{-1} \operatorname{Var}(\epsilon \mid Z, W)\left(\left(I_{n}-\rho W\right)^{-1}\right)^{t}=\sigma^{2}\left(I_{n}-\rho W\right)^{-1}\left(I_{n}-\rho W^{t}\right)^{-1}
\end{aligned}
$$

The spatial weight matrix $\mathrm{W}$ is defined as $W=$ $\left(\omega_{i j}\right)_{1 \leq i, j \leq n}$ where $\omega_{i j}=1$ if the areas $i$ and $j$ are contiguous and $\omega_{i j}=o$ if not. The matrix form is given by:

$$
W=\left[\begin{array}{cccc}
\omega_{11} & \omega_{12} & \ldots & \omega_{1 n} \\
\omega_{21} & \omega_{22} & \ldots & \omega_{2 n} \\
\vdots & \vdots & \ddots & \vdots \\
\omega_{n 1} & \omega_{n 2} & \ldots & \omega_{n n}
\end{array}\right]
$$

By convention, $\omega_{i i}=0$ for all $i=1,2, \ldots, n$. The weight matrix $\mathrm{W}$ is the space version of lag operator in time series [14].
The introduction of this matrix is very important in the spatial linear regression models because it defines the relationships among locations.

The weight matrix $\mathrm{W}$ is typically row-stochastic [15]. It is to say that $\sum_{j=1}^{n} \omega_{i j}=1$ for all $i=1, \ldots, n$. In this case, the spatial lag operator takes the form $y_{L}=W y$ with dimension $n$.

The $i^{\text {th }}$ element of the spatial lag operator $y_{L i}=$ $\sum_{j=1}^{n} \omega_{i j} y_{j}$ is shown as a weighted average of the observations of $y$ in the neighbor of $i$.

For application of this article, we use real data to perform 
the spatial Durbin model. The data comes from the National Agency of Statistics and Demography of Senegal (RGPHAE, 2013). We are interested to the relationship between the rate of divorce and the rate of illiteracy and the average age at marriage in Senegal. The data was collected in 45 departments of the country. In this study, the dependent variable is the rate of divorce and the explanatory variables the rate of illiteracy and the average age at marriage.

$y$ is given by:

Where $\frac{\partial \epsilon}{\partial y}$ is the jacobian matrix.

\section{Results}

Parameter estimation is done here by maximum likelihood procedure. From the equation of $\operatorname{SDM} y=\rho W y+Z \delta+$ $\epsilon, \quad \epsilon \sim N\left(0, \sigma^{2} I_{n}\right)$, we obtain:

$$
\epsilon=y-\rho W y-Z \delta=\left(I_{n}-\rho W\right) y-Z \delta=A y-Z \delta
$$

Where $A=I_{n}-\rho W$. Noting $\theta=\left(\delta^{t}, \sigma^{2}, \rho\right)^{t}$ and using the transformation theorem, the probability density function of

Since $\epsilon \sim N\left(0, \sigma^{2} I_{n}\right)$, we have:

$$
\begin{gathered}
f(\epsilon \mid Z ; \theta)=\frac{1}{(2 \pi)^{\frac{n}{2}}\left|\sigma^{2} I_{n}\right|^{\frac{1}{2}}} \exp \left[-\frac{1}{2}(\epsilon-0)^{t}\left(\sigma^{2} I_{n}\right)^{-1}(\epsilon-0)\right] \\
=\frac{1}{(2 \pi)^{\frac{n}{2}}\left(\sigma^{2}\right)^{\frac{n}{2}}} \exp \left[-\frac{1}{2 \sigma^{2}} \epsilon^{t} \epsilon\right]=(2 \pi)^{-\frac{n}{2}}\left(\sigma^{2}\right)^{-\frac{n}{2}} \exp \left[-\frac{1}{2 \sigma^{2}}(A y-Z \delta)^{t}(A y-Z \delta)\right]
\end{gathered}
$$

We also have:

$$
\left|\frac{\partial \epsilon}{\partial y}\right|=\left|\frac{\partial(A y-Z \delta)}{\partial y}\right|=\left|\frac{\partial A y}{\partial y}-\frac{\partial z \delta}{\partial y}\right|=\left|\frac{\partial A y}{\partial y}\right|=|A|=\left|I_{n}-\rho W\right|
$$

Then the likelihood function of the model is:

$$
L(\theta)=f(y \mid Z ; \theta)=(2 \pi)^{-\frac{n}{2}}\left(\sigma^{2}\right)^{-\frac{n}{2}} \exp \left[-\frac{1}{2 \sigma^{2}}(A y-Z \delta)^{t}(A y-Z \delta)\right]|A|
$$

The log-likelihood function of the model is:

$$
\log (L(\theta))=-\frac{n}{2} \log (2 \pi)-\frac{n}{2} \log \left(\sigma^{2}\right)-\frac{1}{2 \sigma^{2}}(A y-Z \delta)^{t}(A y-Z \delta)+\log (|A|)
$$

To find the maximum likelihood estimate of SDM parameters, we need to maximize equation (9) with respect to $\theta$.

Doing the derivative of equation (9) with respect to $\delta$, we obtain:

$$
\begin{gathered}
\frac{\partial \log (L(\theta))}{\partial \delta}=\frac{\partial}{\partial \delta}\left(-\frac{n}{2} \log (2 \pi)-\frac{n}{2} \log \left(\sigma^{2}\right)-\frac{1}{2 \sigma^{2}}(A y-Z \delta)^{t}(A y-Z \delta)+\log (|A|)\right) \\
=-\frac{1}{2 \sigma^{2}} \frac{\partial}{\partial \delta}\left((A y-Z \delta)^{t}(A y-Z \delta)\right)=-\frac{1}{2 \sigma^{2}}\left[2 \frac{\partial(A y-Z \delta)^{t}}{\partial \delta}(A y-Z \delta)\right] \\
=-\frac{1}{\sigma^{2}} \frac{\partial(-Z \delta)^{t}}{\partial \delta}(A y-Z \delta)=\frac{1}{\sigma^{2}} Z^{t}(A y-Z \delta)=\frac{1}{\sigma^{2}} Z^{t} \epsilon
\end{gathered}
$$

Taking the derivative of equation (9) with respect to $\sigma^{2}$, we obtain:

$$
\begin{gathered}
\frac{\partial \log (L(\theta))}{\partial \sigma^{2}}=\frac{\partial}{\partial \sigma^{2}}\left(-\frac{n}{2} \log (2 \pi)-\frac{n}{2} \log \left(\sigma^{2}\right)-\frac{1}{2 \sigma^{2}}(A y-Z \delta)^{t}(A y-Z \delta)+\log (|A|)\right) \\
=-\frac{n}{2} \frac{\partial \log \left(\sigma^{2}\right)}{\partial \sigma^{2}}-\frac{1}{2} \frac{\partial}{\partial \sigma^{2}}\left(\frac{1}{\sigma^{2}}(A y-Z \delta)^{t}(A y-Z \delta)\right) \\
=-\frac{n}{2 \sigma^{2}}+\frac{1}{2 \sigma^{4}}(A y-Z \delta)^{t}(A y-Z \delta)=-\frac{n}{2 \sigma^{2}}+\frac{1}{2 \sigma^{4}} \epsilon^{t} \epsilon
\end{gathered}
$$

Taking the derivative of equation (9) respect to $\rho$, we obtain:

$$
\frac{\partial \log (L(\theta))}{\partial \rho}=\frac{\partial}{\partial \rho}\left(-\frac{n}{2} \log (2 \pi)-\frac{n}{2} \log \left(\sigma^{2}\right)-\frac{1}{2 \sigma^{2}}(A y-Z \delta)^{t}(A y-Z \delta)+\log (|A|)\right)
$$




$$
\begin{gathered}
=-\frac{1}{2 \sigma^{2}} \frac{\partial}{\partial \rho}\left((A y-Z \delta)^{t}(A y-Z \delta)\right)+\frac{\partial}{\partial \rho} \log (|A|) \\
=-\frac{1}{2 \sigma^{2}}\left((A y-Z \delta)^{t} \frac{\partial(A y-Z \delta)}{\partial \rho}+\frac{\partial(A y-Z \delta)^{t}}{\partial \rho}(A y-Z \delta)\right)+\operatorname{tr}\left(A^{-1} \frac{\partial A}{\partial \rho}\right) \\
=-\frac{1}{2 \sigma^{2}}\left(2(A y-Z \delta)^{t} \frac{\partial(A y-Z \delta)}{\partial \rho}\right)+\operatorname{tr}\left(A^{-1} \frac{\partial A}{\partial \rho}\right)=-\frac{1}{\sigma^{2}}\left((A y-Z \delta)^{t} \frac{\partial(A y)}{\partial \rho}\right)+\operatorname{tr}\left(A^{-1} \frac{\partial A}{\partial \rho}\right) \\
=-\frac{1}{\sigma^{2}}\left((A y-Z \delta)^{t} \frac{\partial\left(I_{n}-\rho W\right) y}{\partial \rho}\right)+\operatorname{tr}\left(A^{-1} \frac{\partial\left(I_{n}-\rho W\right)}{\partial \rho}\right)=-\frac{1}{\sigma^{2}}\left((A y-Z \delta)^{t} \frac{\partial(-\rho W) y}{\partial \rho}\right)+\operatorname{tr}\left(A^{-1} \frac{\partial(-\rho W)}{\partial \rho}\right) \\
=\frac{1}{\sigma^{2}}(A y-Z \delta)^{t} W y+\operatorname{tr}\left(A^{-1}(-W)\right)=\frac{1}{\sigma^{2}} \epsilon^{t} W y-\operatorname{tr}\left(A^{-1} W\right)
\end{gathered}
$$

The complete gradient of the model is now given by:

$$
\nabla_{\theta}=\frac{\partial \log (L(\theta))}{\partial \theta}=\left(\begin{array}{l}
\frac{\partial \log (L(\theta))}{\partial \delta} \\
\frac{\partial \log (L(\theta))}{\partial \sigma^{2}} \\
\frac{\partial \log (L(\theta))}{\partial \rho}
\end{array}\right)=\left(\begin{array}{c}
\frac{1}{\sigma^{2}} Z^{t} \epsilon \\
\frac{1}{2 \sigma^{4}}\left(\epsilon^{t} \epsilon-n \sigma^{2}\right) \\
\frac{1}{\sigma^{2}} \epsilon^{t} W y-\operatorname{tr}\left(A^{-1} W\right)
\end{array}\right)
$$

Parameter estimates are obtained by solving $\nabla_{\theta}=0$. But the real problem is to solve the equation $\frac{1}{\sigma^{2}} \epsilon^{t} W y-\operatorname{tr}\left(A^{-1} W\right)=0$ which is highly non-linear. The solution is to use the concentrated log-likelihood function [12].

Setting $\frac{1}{\sigma^{2}} Z^{t} \epsilon=0$ and solving, we obtain:

$$
Z^{t} \epsilon=Z^{t}(A y-Z \delta)=Z^{t} A y-Z^{t} Z \delta=0 \quad \text { and } \quad \hat{\delta}=\left(Z^{t} Z\right)^{-1} Z^{t} A
$$

Setting also $\frac{1}{2 \sigma^{4}}\left(\epsilon^{t} \epsilon-n \sigma^{2}\right)=0$, we obtain:

$$
\epsilon^{t} \epsilon-n \sigma^{2}=0 \quad \text { and } \quad \hat{\sigma}^{2}=\frac{\hat{\epsilon}^{t} \hat{\epsilon}}{n}=\frac{\left.(A y-Z \widehat{\delta})^{t}(A y-Z \widehat{\delta})\right)}{n}
$$

Equation (10) can be written as:

$$
\hat{\delta}=\left(Z^{t} Z\right)^{-1} Z^{t} A y=\left(Z^{t} Z\right)^{-1} Z^{t}\left(I_{n}-\rho W\right) y=\left(Z^{t} Z\right)^{-1} Z^{t} y-\rho\left(Z^{t} Z\right)^{-1} Z^{t} W y=\widehat{\delta_{0}}-\rho \widehat{\delta_{1}}
$$

Where $\widehat{\delta_{0}}=\left(Z^{t} Z\right)^{-1} Z^{t} y$ and $\widehat{\delta_{1}}=\left(Z^{t} Z\right)^{-1} Z^{t} W y$. Next, define the expressions:

$$
e_{o}=y-Z \widehat{\delta_{0}} \quad \text { and } \quad e_{1}=W y-Z \widehat{\delta_{1}}
$$

We can now write:

$$
\begin{gathered}
\hat{\sigma}^{2}=\frac{\left.(A y-Z \hat{\delta})^{t}(A y-Z \widehat{\delta})\right)}{n}=\frac{\left(A y-Z\left(\widehat{\delta_{0}}-\rho \widehat{\delta_{1}}\right)\right)^{t}\left(A y-Z\left(\widehat{\delta_{0}}-\rho \widehat{\delta_{1}}\right)\right)}{n} \\
=\frac{\left(A y+e_{0}-y+\rho\left(W y-e_{1}\right)\right)^{t}\left(A y+e_{0}-y+\rho\left(W y-e_{1}\right)\right)}{n} \\
=\frac{\left(\left(I_{n}-\rho W\right) y+e_{0}-y+\rho\left(W y-e_{1}\right)\right)^{t}\left(\left(I_{n}-\rho W\right) y+e_{0}-y+\rho\left(W y-e_{1}\right)\right)}{n}=\frac{\left(e_{0}-\rho e_{1}\right)^{t}\left(e_{0}-\rho e_{1}\right)}{n}
\end{gathered}
$$

Now replacing $\delta$ and $\sigma^{2}$ respectively by $\hat{\delta}=\widehat{\delta_{0}}-\rho \widehat{\delta}_{1}$ and $\hat{\sigma}^{2}=\frac{\left(e_{0}-\rho e_{1}\right)^{t}\left(e_{0}-\rho e_{1}\right)}{n}$ in equation (9), we obtain the concentrated log-likelihood function defined as:

$$
\log (L(\rho))_{c}=-\frac{n}{2}-\frac{n}{2} \log (2 \pi)-\frac{n}{2} \log \left[\frac{\left(e_{0}-\rho e_{1}\right)^{t}\left(e_{0}-\rho e_{1}\right)}{n}\right]+\log \left(\left|I_{n}-\rho W\right|\right)
$$

The estimation of the autoregressive parameter $\rho$ is obtained by numerical optimization of the concentrated 
log-likelihood function $\log (L(\rho))_{c}$. However, the computation of $\left|I_{n}-\rho W\right|$ is not simple. To avoid this problem, several methods are proposed in the literature.
We note that $\left|I_{n}-\rho W\right|=\prod_{i=1}^{n}\left(1-\rho \omega_{i}\right) \quad$ where $\omega_{i}, i=1, \ldots, n$ are the eigenvalues of $W[16]$. Therefore, the concentrated log-likelihood function follows as:

$$
\log (L(\rho))_{c}=-\frac{n}{2}-\frac{n}{2} \log (2 \pi)-\frac{n}{2} \log \left[\frac{\left(e_{0}-\rho e_{1}\right)^{t}\left(e_{0}-\rho e_{1}\right)}{n}\right]+\sum_{i=1}^{n} \log \left(1-\rho \omega_{i}\right)
$$

The use of direct sparse matrix algorithms such as Cholesky decompositions or a Monte Carlo estimator in order to compute the $\log$-determinant $\log \left(\left|I_{n}-\rho W\right|\right)$ over a grid of values for the parameter $\rho$ is very helpful in practice $[12,17]$.

Specifically, for a grid of $q$ values in the interval $[0,1)$, we have:

$$
\left(\begin{array}{c}
\log \left(L\left(\rho_{1}\right)\right) \\
\log \left(L\left(\rho_{2}\right)\right) \\
\vdots \\
\log \left(L\left(\rho_{q}\right)\right)
\end{array}\right)=\left(\begin{array}{c}
-\frac{n}{2}-\frac{n}{2} \log (2 \pi)-\frac{n}{2} \log \left[\frac{\left(e_{0}-\rho_{1} e_{1}\right)^{t}\left(e_{0}-\rho_{1} e_{1}\right)}{n}\right]+\log \left(\left|I_{n}-\rho_{1} W\right|\right) \\
-\frac{n}{2}-\frac{n}{2} \log (2 \pi)-\frac{n}{2} \log \left[\frac{\left(e_{0}-\rho_{2} e_{1}\right)^{t}\left(e_{0}-\rho_{2} e_{1}\right)}{n}\right]+\log \left(\left|I_{n}-\rho_{2} W\right|\right) \\
\vdots \\
-\frac{n}{2}-\frac{n}{2} \log (2 \pi)-\frac{n}{2} \log \left[\frac{\left(e_{0}-\rho_{q} e_{1}\right)^{t}\left(e_{0}-\rho_{q} e_{1}\right)}{n}\right]+\log \left(\left|I_{n}-\rho_{q} W\right|\right)
\end{array}\right)
$$

\section{Discussion}

In this section, we give the estimation of parameters of spatial Durbin model described as:

$$
y=\alpha 1_{n}+\rho W y+X_{1} \beta_{1}+X_{2} \beta_{2}+W X_{1} \gamma_{1}+W X_{2} \gamma_{2}+\epsilon
$$

Where:

$y$ is a vector that collects the rate of divorce in the 45 departments of Senegal;

$X_{1}$ is a vector that collects the rate of illiteracy in the 45 departments of Senegal;

$X_{2}$ is a vector that collects the average age at marriage in the 45 departments;

Parameter $\alpha$ is intercept;

$\beta_{1}$ and $\beta_{2}$ are real coefficients parameters without weighting;

$\gamma_{1}$ and $\gamma_{1}$ are spatial lag coefficients of the independent variables;

And $\rho$ is the spatial autoregressive parameter.

We use the R Software to simulate this spatial model. Specially, we use the spdep package [18].

The results are shown in the table below:

Table 1. Parameter estimation by SDM.

\begin{tabular}{lccccccc}
\hline Parameters & $\boldsymbol{\alpha}$ & $\boldsymbol{\beta}_{\mathbf{1}}$ & $\boldsymbol{\beta}_{\mathbf{2}}$ & $\boldsymbol{\gamma}_{\mathbf{1}}$ & $\boldsymbol{\gamma}_{\mathbf{2}}$ & $\boldsymbol{\rho}$ & $\boldsymbol{\sigma}$ \\
\hline Estimate & -9.89 & -0.05 & 0.12 & 0.09 & 0.27 & 0.40 & 0.16 \\
P-value & 0.05 & 0.008 & 0.03 & 0.0006 & 0.04 & 0.04 & 0.01 \\
\hline
\end{tabular}

It appears that all parameters are significant except the intercept. We note also that the departments that are close are more similar than the distant departments in relation to divorce data $(\rho=0.4)$.

We can also compute direct and indirect effects on divorce [12].

The reduced form of the SDM model is:

$$
y=\left(I_{n}-\rho W\right)^{-1} Z \delta+\left(I_{n}-\rho W\right)^{-1} \epsilon
$$

Where $S_{r}(W)=A^{-1}\left(I_{n} \beta_{r}+W \delta_{r}\right)$, and $X_{r}=\left(\begin{array}{c}x_{r 1} \\ x_{r 2} \\ \vdots \\ x_{r n}\end{array}\right)$.

The indirect effect is defined as $\frac{\partial \mathbb{E}\left(y_{i}\right)}{\partial x_{j r}}=S_{r}(W)_{i j}$;

The direct effect is given by $\frac{\partial \mathbb{E}\left(y_{i}\right)}{\partial x_{i r}}=S_{r}(W)_{i i}$.

The results of direct and indirect effects are shown in the table below:

Table 2. Impact measures of SDM.

\begin{tabular}{llll}
\hline Effect & Direct & Indirect & Total \\
\hline Illiteracy & -0.04 & 0.12 & 0.08 \\
Average age at marriage & 0.16 & 0.49 & 0.65 \\
\hline
\end{tabular}

We note that an increase of one point of the rate of illiteracy leads to an increase of 0.08 point of the rate of divorce in Senegal. Similarly, an increase of one point of the average age at marriage will increase 0.65 point to the rate of divorce.

\section{Conclusion}

This paper has attempted to model the spatial spillovers of divorce in Senegal using the spatial Durbin model. The theoretical results show that the log-likelihood function of the SDM model is:

$$
\begin{aligned}
\log (L(\theta))= & -\frac{n}{2} \log (2 \pi)-\frac{n}{2} \log \left(\sigma^{2}\right)-\frac{1}{2 \sigma^{2}}(A y \\
& -Z \delta)^{t}(A y-Z \delta)+\log (|A|)
\end{aligned}
$$

Maximization of this log-likelihood function leads to the estimators of parameters $\delta$ and $\sigma^{2}$ like:

$$
\hat{\delta}=\left(Z^{t} Z\right)^{-1} Z^{t} A y \text { and } \hat{\sigma}^{2}=\frac{\left.(A y-Z \widehat{\delta})^{t}(A y-Z \widehat{\delta})\right)}{n}
$$

The estimation of the autoregressive parameter $\rho$ is obtained by numerical optimization of the concentrated 
log-likelihood function given by:

$$
\begin{gathered}
\log (L(\rho))_{c}=-\frac{n}{2}-\frac{n}{2} \log (2 \pi)-\frac{n}{2} \log \left[\frac{\left(e_{0}-\rho e_{1}\right)^{t}\left(e_{0}-\rho e_{1}\right)}{n}\right]+ \\
\log \left(\left|I_{n}-\rho W\right|\right)
\end{gathered}
$$

The empirical results of the SDM model applied to divorce data show that the rate of illiteracy and the average age at marriage have significant association with the rate of divorce in Senegal. In fact, an increase of one point of the rate of illiteracy will increase 0.08 point the rate of divorce in Senegal In the same way, an increase of one point of the average age at marriage leads to an increase of 0.65 point to the rate of divorce in Senegal. The empirical results show also that the departments that are close are more similar than the distant departments in relation to the divorce data

\section{References}

[1] LeSage, J. P., and Pace, R. K. (2017). Spatial econometrics Monte Carlo studies: raising the bar. Empirical Economics, 55(1), 17-34

[2] Doran, J. and Fingleton, B. (2018). US Metropolitan Area Resilience: Insights from dynamic spatial panel estimation, Environment and Planning A: Economy and Space, 50(1), pp. 111-132.

[3] Elhorst, J. P (2014). Spatial Econometrics: From Cross-Sectional Data to Spatial Panels. Springer Briefs in Regional Science.

[4] LeSage, J. P. (2014). What Regional Scientists Need to Know about Spatial Econometrics. The Review of Regional Studies, 44(1).

[5] Golgher, A. B., and Voss, P. R. (2016). How to interpret the coefficients of spatial models: Spillovers, direct and indirect effects. Spatial Demography, 4(3), 175-205.

[6] Koroglu, M., and Sun, Y. (2016). Functional-Coefficient Spatial Durbin Models with Nonparametric Spatial Weights: An Application to Economic Growth. Econometrics, 4(1), 6.
[7] Feng, Z., \& Chen, W. (2018). Environmental Regulation, Green Innovation, and Industrial Green Development: An Empirical Analysis Based on the Spatial Durbin Model. Sustainability, 10(1), 223.

[8] Lee, L., and Yu, J. (2015). Identification of Spatial Durbin Panel Models. Journal of Applied Econometrics, 31(1), 133162.

[9] Bekti R. D., Rahayu A. and Sutikno (2013). Maximum likelihood estimation for spatial Durbin model. Journal of Mathematics and Statistics 9 (3): 169-174, 2013.

[10] Fingleton B. and LeGallo J. (2012). Endogéneité et autocorrélation spatiale: quelle utilité pour le modèle de Durbin? Revue d'économie régionale et urbaine, 2012/1 (février), pp. 3-17.

[11] Seya, H., Tsutsumi M. and Yamagata Y. (2012). Income convergence in Japan: A Bayesian spatial Durbin model approach. Econ. Model., 29: 60-71.

[12] LeSage, J. P. and Pace R. K. (2009). Introduction to Spatial Econometrics. CRC Press Taylor \& Francis Group, Boca Raton.

[13] Anselin, L. (1988). Spatial Econometrics: Methods and Models, Kluwer Academic Publishers, Dorddrecht.

[14] Andrienko N. and Andrienko G. (2006). Exploratory analysis of spatial and temporal data, a systematic approach. Springer-Verlag, Heidelberg, Germany.

[15] LeSage, J. P. and Pace R. K. (2014). The biggest myth in spatial econometrics. Econometrics, 2, pp. 217-249.

[16] Ord, J. K. (1975). Estimation Methods for Models of Spatial Interaction. Journal of the American Statistical Association, Volume 70, pp. 120-126.

[17] Barry, R. and Pace R. K. (1999). A Monte Carlo Estimator of the Log Determinant of Large Sparse Matrices. Linear Algebra and its Applications, Volume 289, pp. 41-54.

[18] Bivand R. S., Pebesma E., Gomez-Rubio V. (2013). Applied spatial data analysis with R, Second edition. Springer, NY. 